Helena Pawlak, Faculty of Mathematics and Informatics, Łódź University, Banacha 22, 90-238 Łódź, Poland. email: rpawlak@math.uni.lodz.pl

Ryszard J. Pawlak, Faculty of Mathematics and Informatics, Łódź University, Banacha 22, 90-238 Łódź, Poland. email: rpawlak@math.uni.lodz.pl

\title{
FIRST-RETURN LIMITING NOTIONS AND RINGS OF SHARKOVSKY FUNCTIONS
}

\begin{abstract}
In this paper we apply some elements of real analysis theory to the distinguishing of the certain subclass of the class of functions possessing Sharkovsky property. The main aim of this is connected with the answer to the following question: what kind of assumption should we impose on Sharkovsky function $f$ in order to have that there exists a ring of functions possessing Sharkovsky property containing $f$ ?
\end{abstract}

Introduction. Dynamical systems have been considered almost exclusively in relationship with continuous functions and even smaller classes. But some considerations lead us to the case when the basis of considerations are, for example, almost continuous functions (some question raised by W. Transue, which is cited in [21]), discontinuous derivatives and other classes of functions (some commentary connected with this topic can be found in [32]). Consequently, recently a lot of papers are connected with dynamical systems generated by Darboux-like functions (e.g. [7], [21], [26], [29], [30], [31], [33]).

The main aim of this paper is to consider some problems connected with the existence of rings of functions possessing Sharkovsky property.

The first part of this paper deals with basic notation and notions which are used in this paper. The second section is devoted to the introduction of a notion convenient for further considerations. The basis of these considerations is first-return limiting theory, which has been considered among others in papers

Mathematical Reviews subject classification: Primary: 26A18, 37E15; Secondary: 26A15, $54 \mathrm{C} 40,54 \mathrm{H} 25$

Key words: od-set, Darboux function, first-return continuity, $\mathcal{S}$-function, Sharkovsky function, ring of functions, trajectory, property $\mathcal{J}$, family substituted by a subfamily

Received by the editors November 29, 2008

Communicated by: Emma D'Aniello 
of American mathematicians [9], [10], [11], [12]. The notion of $\mathcal{S}$-functions (the third part of this paper) is based on first-return language, which permits us to consider some subclasses of Sharkovsky functions consisting of functions for which one can construct suitable rings.

\section{Preliminaries.}

We will use mostly standard definitions and notations (see [1], [2], [3], [4], [13], [14], [15]). In particular by the letter $\mathbb{Z}(\mathbb{R})$ we will denote the set of integer (real) numbers.

The closure and interior of a set $A$ we denote by $\bar{A}$ and $\operatorname{Int}(A)$, respectively. The cardinality of a set $A$ we will denote by the symbol card $(A)$.

We will consider the behaviour of some real functions defined on the real line $\mathbb{R}$. Let $f$ be a real function. By the symbol $B(f, \epsilon)$ we shall denote the open ball with a centre at $f$ and a radius $\epsilon>0$ in the metric space of real functions equipped with the metric $\rho(f, g)=\min \left(1, \sup _{x}|f(x)-g(x)|\right)$.

A set $H \subset \mathbb{R}$ is called an od-set if $H$ is an open subset of $\mathbb{R}$ and $\bar{H}=\mathbb{R}$.

The idea of the notions below derives from [8], [9], [11], [12], [15], [22]: Let $H$ be an od-set in $\mathbb{R}$. By $H$-trajectory we mean any sequence $\left\{d_{n}\right\} \subset H$ of distinct points such that $\left\{d_{n}: n=1,2, \ldots\right\}$ is a dense set in $H$. For a given open set $V \subset H$, and $H$-trajectory $\left\{d_{n}: n=1,2, \ldots\right\}, r_{\left\{d_{n}\right\}}(V)$ (or shortly $r(V)$, if the $H$-trajectory is fixed) will be the first element of the sequence $\left\{d_{n}\right\}$ in $V$.

Let $H$ be an od-set in $\mathbb{R}$ and $\left\{d_{n}\right\} \subset H$ be a fixed $H$-trajectory. For $x \in \mathbb{R}$ the left first return path to $x$ based on $\left\{d_{n}\right\}, P_{x}^{l}=\left\{t_{k}: k=1,2, \ldots\right\}$ is defined recursively via

$$
t_{1}=r((-\infty, x)) ; \quad t_{k+1}=r\left(\left(t_{k}, x\right)\right) .
$$

For $x \in \mathbb{R}$ the right first return path to $x$ based on $\left\{d_{n}\right\}, P_{x}^{r}=\left\{s_{k}: k=1,2, \ldots\right\}$ is defined analogously:

$$
s_{1}=r((x,+\infty)) ; \quad s_{k+1}=r\left(\left(x, s_{k}\right)\right) .
$$

A function $f: \mathbb{R} \rightarrow \mathbb{R}$ is first return continuous from the left (right) at $x$ with respect to the $H$-trajectory $\left\{d_{n}\right\}$ provided that

$$
\lim _{\substack{t \rightarrow x \\ t \in P_{x}^{l}}} f(t)=f(x)\left(\lim _{\substack{t \rightarrow x \\ t \in P_{x}^{r}}} f(t)=f(x)\right) .
$$


We say that $f: \mathbb{R} \rightarrow \mathbb{R}$ is a first return continuous function at $x$ with respect to the $H$ - trajectory $\left\{d_{n}\right\}$ provided it is both left and right first return continuous at $x$ with respect to the $H$ - trajectory $\left\{d_{n}\right\}$.

Let $\left\{d_{n}\right\}$ be a fixed $H$-trajectory. A function $f: \mathbb{R} \rightarrow \mathbb{R}$ is an $\left(H,\left\{d_{n}\right\}\right)$-first return continuous function $\left(f \in \operatorname{FRC}\left(H,\left\{d_{n}\right\}\right)\right)$ if it is first return continuous at each point $x \in H$ (with respect to the $H$-trajectory $\left\{d_{n}\right\}$ ) and for any component $(a, b)$ of the set $H, f$ is first return continuous from the right (left) at $a(b)$ with respect to the $H$-trajectory $\left\{d_{n}\right\}$.

If $H=\mathbb{R}$, then we will write shortly trajectory, first return continuity, etc.

If $A, B$ are subsets of the domain of $f$, then $f \uparrow A$ denotes the restriction of $f$ to $A$. We say that a set $A f$-covers a set $B$ (denoted by $A \underset{f}{\longrightarrow} B$ ), if $B \subset f(A)$.

Let $H$ be an od-set. We say that a set $H f$-replaces $\mathbb{R}$ (denoted by $H \underset{f-r}{\longrightarrow} \mathbb{R}$ ) if for any nondegenerated interval $[\alpha, \beta] \subset \mathbb{R}$ there exists $(a, b) \subset[\alpha, \beta] \cap H$ such that $[a, b] \underset{f}{\longrightarrow} f([\alpha, \beta])$.

Let $f$ be a function. Then $f^{0}(x)=x$, and $f^{n}(x)=f\left(f^{n-1}(x)\right)$, if $n>0$. We say that a set $A$ is $f$-invariant, if $f(A) \subset A$.

A point $x$ such that $f^{M}(x)=x$ but $f^{n}(x) \neq x$, for $n \in\{1,2, \ldots, M-1\}$ is called a periodic point of $f$ of a prime period $M$. The set of all periodic points of $f$ of a prime period $M$ we will denote by $\operatorname{Per}_{M}(f)$.

The graph of a function $f$ we shall denote by $\Gamma(f)$. Let us denote by $i d_{A}$ the identical function defined on $A$. If $\mathcal{F}$ is a family of functions mapping $\mathbb{R}$ into $\mathbb{R}$, then we shall denote $\mathcal{F}^{c}=\left\{f_{1} \circ f_{2} \circ \ldots \circ f_{n}: f_{1}, f_{2}, \ldots, f_{n} \in \mathcal{F}, n \geq 1\right\}$.

Let $\mathcal{D}\left(\mathcal{B}_{1}\right)$ denote the class of all Darboux functions; i.e. functions having Darboux property or, in other words, intermediate value property ([4]) (functions in Baire class 1). If we wish to consider the intersection of two classes, we shall write them next to each other e.g. $\mathcal{D} \mathcal{B}_{1}$ consists of all Darboux functions in Baire class 1.

Suppose we are given a countable cover $\left\{A_{z}\right\}_{z \in \mathbb{Z}}$ of some space $X$ and a family of compatible functions $\left\{f_{z}\right\}$ mapping $A_{z}$ into $\mathbb{R}$ (i.e. $f_{z_{1}}\left\lceil A_{z_{1}} \cap A_{z_{2}}=\right.$ $f_{z_{2}}\left\lceil A_{z_{1}} \cap A_{z_{2}}\right.$, for each integers $\left.z_{1}, z_{2}\right)$. Let us put $f(x)=f_{z}(x)$, for $x \in A_{z}$. Then we have defined a function $f: X \longrightarrow \mathbb{R}$, which is called combination of the functions $\left\{f_{z}\right\}$ and is denoted by $\nabla_{z=-\infty}^{\infty} f_{z}$.

Let us proceed to the definitions and the notations connected with Sharkovsky property. At first, we shall consider the following Sharkovsky ordering of the set of all positive integers.

$$
\begin{gathered}
3 \prec 5 \prec 7 \prec \ldots \prec 2 \cdot 3 \prec 2 \cdot 5 \prec 2 \cdot 7 \prec \ldots \prec 2^{2} \cdot 3 \prec 2^{2} \cdot 5 \prec \ldots \\
\ldots \prec 2^{3} \prec 2^{2} \prec 2 \prec 2^{0}=1 .
\end{gathered}
$$


We shall say that $f$ is Sharkovsky function (or $f$ possesses Sharkovsky property) provided that if $\operatorname{Per}_{M}(f) \neq \emptyset$ and $M \prec K$, then $\operatorname{Per}_{K}(f) \neq \emptyset$.

In the next definitions the addition of index numbers is modulo $M$ (i.e. $M+1=1)^{1}$. Let $\left(I_{1}, I_{2}, \ldots, I_{M}\right)$ be a finite sequence of continuums $\left(I_{i} \subset \mathbb{R}\right.$, for $i=1,2, \ldots)$ and let $f_{1}, f_{2}, \ldots, f_{M}: \mathbb{R} \longrightarrow \mathbb{R}$. We say that $\left(I_{1}, I_{2}, \ldots, I_{M}\right)$ is an $\left(f_{1}, f_{2}, \ldots, f_{M}\right)$-cycle if

$$
I_{1} \underset{f_{1}}{\longrightarrow} I_{2} \underset{f_{2}}{\longrightarrow} I_{3} \underset{f_{3}}{\longrightarrow} \ldots \underset{f_{M-1}}{\longrightarrow} I_{M} \underset{f_{M}}{\longrightarrow} I_{M+1}=I_{1} .
$$

If $f_{1}=f_{2}=\ldots=f_{M}=f$, then we say that an $\left(f_{1}, f_{2}, \ldots, f_{M}\right)$-cycle $\left(I_{1}, I_{2}, \ldots\right.$, $\left.I_{M}\right)$ is an $(f)$-cycle.

If $x_{0} \in I_{1}$ is a point such that

$$
\left(f_{i} \circ f_{i-1} \circ \ldots \circ f_{1}\right)\left(x_{0}\right) \in I_{i+1}, \text { for } i \in\{1,2, \ldots, M\},
$$

then we say that $x_{0}$ is associated with an $\left(f_{1}, f_{2}, \ldots, f_{M}\right)$-cycle $\left(I_{1}, I_{2}, \ldots, I_{M}\right)$.

We say $([33])$ that a family of functions $\mathcal{F}$ has the property $\mathcal{J}$ if for any $\left(f_{1}, f_{2}, \ldots, f_{M}\right)$-cycle $\left(I_{1}, I_{2}, \ldots, I_{M}\right)\left(f_{1}, f_{2}, \ldots, f_{M} \in \mathcal{F}\right)$, there exists a point $x_{0}$ associated with this cycle and such that $\left(f_{M} \circ f_{M-1} \circ \ldots \circ f_{1}\right)\left(x_{0}\right)=x_{0}$.

We say that a family of functions $\mathcal{F}$ has the property $\mathcal{J}_{1}$ if for any $(f)$ cycle $\left(I_{1}, I_{2}, \ldots, I_{M}\right)(f \in \mathcal{F})$, there exists a point $x_{0}$ associated with this cycle and such that $f^{M}\left(x_{0}\right)=x_{0}$ (in the case of continuous functions defined on a compact interval the property $\mathcal{J}_{1}$ is connected with the so called Itinerary Lemma).

From [2] we adopt the idea of the next notion. For our needs we supplement this definition with the condition (5). We say that a family $\mathcal{R}$ of real functions $f$ is an $\mathcal{A S}$-ring of functions if

(1) if $f, g \in \mathcal{R}$, then $\max (f, g), \min (f, g) \in \mathcal{R}$,

(2) $\mathcal{R}$ contains all constants,

(3) if $f, g \in \mathcal{R}$, then $f+g, f \cdot g \in \mathcal{R}$,

(4) the uniform limit of a sequence $\left\{f_{n}\right\} \subset \mathcal{R}$ belongs to $\mathcal{R}$,

(5) if $f \in \mathcal{R}$, then $f$ is Sharkovsky function.

\footnotetext{
${ }^{1}$ In the whole paper, if we have a sequence consisting of $m$ elements, numbered from 1 to $M$, then we accept $M+1=1$ (the addition of index numbers is modulo $M$ ).
} 


\section{$2 \mathcal{S}$-functions.}

In this part of the paper we will introduce the class of $\mathcal{S}$-functions which has its origin in the theory of dynamical systems ([9], [10]). It is interesting to note that this class contains several important families of functions (for example the class of all continuous functions, derivatives, approximately continuous functions, etc.).

Definition 1. Let $H$ be an od-set in $\mathbb{R}$ and $\left\{d_{n}\right\} \subset H$ be a fixed $H$-trajectory. We will call $f: \mathbb{R} \rightarrow \mathbb{R}$ an $\mathcal{S}\left(H,\left\{d_{n}\right\}\right)$-function $\left(f \in \mathcal{S}\left(H,\left\{d_{n}\right\}\right)\right)$ if $H \underset{f-r}{\longrightarrow} \mathbb{R}$ and $f \in \operatorname{FRC}\left(H,\left\{d_{n}\right\}\right)$.

Definition 2. We say that $f: \mathbb{R} \rightarrow \mathbb{R}$ is an $\mathcal{S}$-function $(f \in \mathcal{S})$ provided that there exists an od-set $H$ and an $H$-trajectory $\left\{d_{n}\right\}$ such that $f \in \mathcal{S}\left(H,\left\{d_{n}\right\}\right)$.

Lemma 2.1. Let $f \in \operatorname{FRC}\left(H,\left\{d_{n}\right\}\right)$, for some od-set $H$ and $H$-trajectory $\left\{d_{n}\right\}$. Moreover, let $\left\{d_{m_{n}}\right\} \subset(a, b) \subset H$ be a subsequence of sequence $\left\{d_{n}\right\}$ consisting of all terms belonging to $(a, b)$. Then

$f \uparrow[a, b]$ is first return continuous at $x$ with respect to $\left\{d_{n}\right\}$

if and only if $f$ is first return continuous at $x$ with respect to $\left\{d_{m_{n}}\right\}$,

for $x \in[a, b]$.

Proof. ${ }^{2}$ Let us establish some notation:

$P_{x}^{l \mathbb{R}}=\left\{t_{k}\right\}$ will denote the left first return path to $x$ based on $\left\{d_{n}\right\}$.

$P_{x}^{l T}=\left\{\tau_{\mu}\right\}$ will denote the left first return path to $x$ based on $\left\{d_{m_{n}}\right\}$.

Put $k_{0}=\min \left\{k: t_{k} \in(a, x)\right\}$. Let us notice that $t_{k_{0}}=\tau_{1}$. From this we conclude that

$$
P_{x}^{l \mathbb{R}} \cap\left(t_{k_{0}}, x\right)=P_{x}^{l T} \cap\left(\tau_{1}, x\right) .
$$

This finishes the proof for the first return continuity from the left.

In a similar way one can prove our claim for the first return continuity from the right.

We will show that the family $\mathcal{S}$ (i.e. the set of all $\mathcal{S}$-functions) is a subfamily of $\mathcal{D}$ (Theorem 2.2) and it is useful to characterize the class as $\mathcal{D} \mathcal{B}_{1}$ (Proposition 2.3). So, the class $\mathcal{S}$ is an intermediate family between $\mathcal{D} \mathcal{B}_{1}$ and $\mathcal{D}\left(\mathcal{D} \mathcal{B}_{1} \subset \mathcal{S} \subset \mathcal{D}\right)$. It seems to be interesting that $\mathcal{S}$ contains some nonmeasurable (in the Lebesgue sense) functions (suitable examples are very simple).

\footnotetext{
${ }^{2}$ The proof follows also from ([22], Lemma 1.17)
} 
But for our considerations it is important to remark that the class $\mathcal{S}$ has interesting properties connected with the problem of generalizing Sharkovsky's theorem.

As mentioned above, we start the discussion about $\mathcal{S}$-functions by proving theorems connected with Darboux property.

Theorem 2.2. If $f: \mathbb{R} \longrightarrow \mathbb{R}$ is an $\mathcal{S}$-function, then $f$ is a Darboux function.

Proof. Let $[\alpha, \beta]$ be an arbitrary closed interval in $\mathbb{R}$. Since $f$ is an $\mathcal{S}$ function then there exists an od-set $H$ and $H$-trajectory $\left\{d_{n}\right\}$ such that $f \in$ $\mathcal{S}\left(H,\left\{d_{n}\right\}\right)$. Moreover, there is an open interval $(a, b) \subset[\alpha, \beta] \cap H$ such that $[a, b] \underset{f}{\longrightarrow} f([\alpha, \beta])$. Therefore, $f([a, b])=f([\alpha, \beta])$. Utilizing Lemma 2.1 and Theorem 3 from [10], we have that $f([a, b])$ is a connected set.

It is not hard to verify that there are a lot of properties of $\mathcal{S}$-functions close to the well known properties of continuous functions. The next proposition is an example of this situation.

Proposition 2.3. Let $f: \mathbb{R} \rightarrow \mathbb{R}$. Then $f \in \mathcal{D B}_{1}$ if and only if there exists an od-set $H$ with $\operatorname{card}(\mathbb{R} \backslash H) \leq \aleph_{0}$ and some $H$-trajectory $\left\{d_{n}\right\}$ such that $f \in \mathcal{S}\left(H,\left\{d_{n}\right\}\right)$.

Proof. Necessity. Suppose that $f$ is Darboux function in Baire class one. Let $H=\mathbb{R}$. Note that $f=\nabla_{i=-\infty}^{\infty} f\left\lceil[i, i+1]\right.$ and $f_{i}=f\left\lceil[i, i+1] \in \mathcal{D B}_{1}([i, i+1])\right.$ for any $i \in \mathbb{Z}$. According to Theorem 3 in [10] (if need be, we can translate the domain) we conclude that there exists a dense (in $[i, i+1])$ sequence $\left\{d_{n}^{i}\right\}_{n=0}^{\infty} \subset(i, i+1)$ such that $f_{i}$ is first return continuous with respect to $\left\{d_{n}^{i}\right\}_{n=0}^{\infty}$.

First we will define two sequences $\left\{d_{n}^{*}\right\}_{n=0}^{\infty}$ and $\left\{d_{n}^{* *}\right\}_{n=1}^{\infty}$ in the following way $^{3}$ :

$$
\begin{gathered}
d_{0}^{*}=d_{0}^{0} ; d_{1}^{*}=d_{1}^{0} ; d_{2}^{*}=d_{0}^{1} ; d_{3}^{*}=d_{2}^{0} ; d_{4}^{*}=d_{1}^{1} ; d_{5}^{*}=d_{0}^{2} ; d_{6}^{*}=d_{3}^{0} ; \ldots \\
d_{1}^{* *}=d_{0}^{-1} ; d_{2}^{* *}=d_{1}^{-1} ; d_{3}^{* *}=d_{0}^{-2} ; d_{4}^{* *}=d_{2}^{-1} ; d_{5}^{* *}=d_{1}^{-2} ; d_{6}^{* *}=d_{0}^{-3} ; \ldots
\end{gathered}
$$

Finally let $\left\{d_{n}\right\}$ be a sequence such that $d_{2 n+1}=d_{n}^{*}(n=0,1,2, \ldots)$ and $d_{2 n}=d_{n}^{* *}(n=1,2, \ldots)$. It is not hard to verify that $\left\{d_{n}\right\}$ is an $H$-trajectory.

\footnotetext{
${ }^{3}$ The sequence $\left\{d_{n}^{*}\right\}_{n=0}^{\infty}$ is formed by successive finite sequences such that the sum of lower and upper indexes are fixed natural numbers: $1,2, \ldots$; the sequence $\left\{d_{n}^{* *}\right\}_{n=0}^{\infty}$ is formed by successive finite0 sequences such that the sum of lower index and absolute value of upper index are fixed natural numbers: $1,2, \ldots$
} 
The method of construction of $\left\{d_{n}\right\}$ assures that we have

$$
\begin{gathered}
\text { if } i \in \mathbb{Z} \text { and } d_{n_{1}}^{i}=d_{m_{1}} \text { and } d_{n_{2}}^{i}=d_{m_{2}}, \text { then } \\
n_{1}<n_{2} \text { if and only if } m_{1}<m_{2} .
\end{gathered}
$$

Now, we shall show that $f$ is an $\left(H,\left\{d_{n}\right\}\right)$-first return continuous function. Let $x_{0} \in \mathbb{R}$. Then there exists $i_{0} \in \mathbb{Z}$ such that $x_{0} \in\left[i_{0}, i_{0}+1\right)$. Since $f_{i_{0}}$ is first return continuous from the right at $x_{0}$, then (by Lemma 2.1) $f$ is first return continuous from the right at $x_{0}$. So, the statement $f \in \operatorname{FRC}\left(H,\left\{d_{n}\right\}\right)$ is obvious. Finally, we can note that $f \in \mathcal{S}\left(H,\left\{d_{n}\right\}\right)$.

Sufficiency. According to Theorem 2.2 and our assumption $\operatorname{card}(\mathbb{R} \backslash H) \leq$ $\aleph_{0}, f \in \mathcal{D} \mathcal{B}_{1}$.

The above theorem and classical results connected with the class $\mathcal{D} \mathcal{B}_{1}$ show that the family $\mathcal{S}$ contains many important classes of functions. For example: the family of all derivatives, the family of all approximately continuous functions etc. are subfamilies of $\mathcal{S}([4])$. On the other hand, this family is very interesting for our considerations, connected with Sharkovsky's theorem.

\section{Rings of Sharkovsky Functions.}

In [1] one can read: When studying properties of cycles of maps, it is desirable to prove that a given property is stable, in the sense that if a map $f$ has this property then small perturbations of $f$ (maps close to $f$ in an appropriate topology) also have this property. The words "small perturbations" can be interpreted as some operations performed on functions (addition, multiplication, lattice operations, etc.). This lead us to the considerations connected with rings of functions. The earlier results obtained in the area of dynamical systems generated by discontinuous functions (contained, for example, in the papers [32], [33], [21], [26], [7]) suggest problems concentrating around the stability of the Sharkovsky property and, consequently, lead us to the questions connected with existence of rings of Sharkovsky functions. This topic is also connected with the widely considered (in the theory of real functions) problems concerning some operations performed on Darboux-like functions (see, for example, [4], [5], [6], [16], [17], [18], [19], [20], [23], [24], [25], [27], [28], etc.).The starting point for these considerations is a remark that even simple operations performed on almost continuous functions lead one out of this class even if both functions considered are discontinuous at one and the same point.

It is easy to construct a discontinuous Sharkovsky function $f$ such that there is no ring consisting of Sharkovsky functions containing $f$. In fact, put $f(x)=1$, if $x \notin\{-1,0\}, f(-1)=0, f(0)=-1$ (in this case $-f$ is not 
Sharkovsky function). Simultaneously, an arbitrary modification of the value of $f$ at 1 , gives a function which is not a Sharkovsky function. The above considerations lead us to the following questions: What kind of conditions should we impose on a family of Sharkovsky functions $\mathcal{F}$ in order to have that for each $f \in \mathcal{F}$ there exists a ring $\mathcal{R} \subset \mathcal{F}$ such that $f \in \mathcal{R}$ ?

The answer to this question is contained in Theorem 3.3.

For our considerations it is very useful to introduce the following notions $([30])$.

Definition 3. We say that an $(f)$-cycle $\left(J_{1}, J_{2}, \ldots, J_{M}\right)$ predominates over $\left(f_{1}, f_{2}, \ldots, f_{M}\right)$-cycle $\left(I_{1}, I_{2}, \ldots, I_{M}\right)$ if for each $i \in\{1,2, \ldots, M\}$ there exists a homeomorphic embedding $\xi_{i}: J_{i} \rightarrow I_{i}$ such that

$$
\left(f_{i} \circ \ldots \circ f_{1}\right)\left(\xi_{1}(x)\right)=\xi_{i+1}\left(f^{i}(x)\right)
$$

for each point $x$ associated with the $(f)$-cycle $\left(J_{1}, J_{2}, \ldots, J_{M}\right)$.

Definition 4. We say that a family of functions $\mathcal{F}$ is substituted by a family $\mathcal{F}_{1} \subset \mathcal{F}$ if for any natural number $M$ and an arbitrary $\left(f_{1}, f_{2}, \ldots, f_{M}\right)$-cycle $\left(I_{1}, I_{2}, \ldots, I_{M}\right)$, where $f_{1}, \ldots, f_{M} \in \mathcal{F}$, there exists an $(f)$-cycle $\left(J_{1}, J_{2}, \ldots, J_{M}\right)$ predominating over $\left(f_{1}, f_{2}, \ldots, f_{M}\right)$-cycle $\left(I_{1}, I_{2}, \ldots, I_{M}\right)$ such that $f \in \mathcal{F}_{1}$.

Lemma 3.1. [30] Let us suppose that $\mathcal{F} \subset \mathcal{D}$ is a family of functions substituted by family $\mathcal{D} \mathcal{B}_{1}$. Then each function $f \in \mathcal{F}^{c}$ is a Sharkovsky function.

The detailed proof will appear in [30], Theorem 2.3.

Now, let us proceed to the two theorems that are, in particular, solutions of our main problem.

Lemma 3.2. The family $\mathcal{S}^{c}$ consists of Sharkovsky functions.

Proof. According to Theorem 2.2, Proposition 2.3 and Lemma 3.1 it is sufficient to show that (of course, any family of functions possessing connected graph which is a $G_{\delta}$ set has the property $\left.\mathcal{J}_{1}([33])\right)$

the family $\mathcal{S}$ is substituted by a family $\mathcal{D} \mathcal{B}_{1}$.

Let $\left(I_{1}, I_{2}, \ldots, I_{M}\right)$ be a fixed $\left(f_{1}, f_{2}, \ldots, f_{M}\right)$-cycle; i.e.

$$
I_{1} \underset{f_{1}}{\longrightarrow} I_{2} \underset{f_{2}}{\longrightarrow} \underset{f_{M-1}}{\longrightarrow} I_{M} \underset{f_{M}}{\longrightarrow} I_{1} .
$$


Let $H_{i}$ be a fixed od-set and let $\left\{d_{n}^{i}\right\}$ be a fixed $H_{i}$-trajectory such that $f_{i} \in \mathcal{S}\left(H_{i},\left\{d_{n}^{i}\right\}\right)$ (for $\left.i=1,2, \ldots, M\right)$. Thus for any $i \in\{1,2, \ldots, M\}$ there exists an interval $\left(a_{i}, b_{i}\right) \subset I_{i} \cap H_{i}$ such that $\left[a_{i}, b_{i}\right] \underset{f_{i}}{\longrightarrow} f_{i}\left(I_{i}\right)$. So, we have

$$
\left[a_{1}, b_{1}\right] \underset{f_{1}}{\longrightarrow}\left[a_{2}, b_{2}\right] \underset{f_{2}}{\longrightarrow} \ldots \underset{f_{M-1}}{\longrightarrow}\left[a_{M}, b_{M}\right] \underset{f_{M}}{\longrightarrow}\left[a_{1}, b_{1}\right] .
$$

The rest of the proof runs as in the proof of the statement contained in paper [30] that the family $\mathcal{P}_{\mathcal{C}}$ is substituted by $\mathcal{C}$. This accounts, for presenting only the sketch of the next part of the proof.

Let $\left\{\left[a_{i}^{\prime}, b_{i}^{\prime}\right]\right\}_{i=1}^{M}$ be a sequence of disjoint intervals with the length $b_{i}-a_{i}$ and put $\delta_{i}=a_{i}^{\prime}-a_{i}$.

Let $\xi_{i}:\left[a_{i}^{\prime}, b_{i}^{\prime}\right] \rightarrow I_{i}$ be a homeomorphic embedding defined by the formula

$$
\xi_{i}(x)=x-\delta_{i}, i=1,2, \ldots, M .
$$

Let us define a function $f: \mathbb{R} \rightarrow \mathbb{R}$ in the following way: $f(x)=f_{i}\left(\xi_{i}(x)\right)+$ $\delta_{i+1}$, for $x \in\left[a_{i}^{\prime}, b_{i}^{\prime}\right](i=1,2, \ldots, M)$ and $f$ is a linear function on the closure of any component of the complement of $\bigcup_{i=1}^{M}\left[a_{i}^{\prime}, b_{i}^{\prime}\right]$. It is easy to see that $f \in \mathcal{D} \mathcal{B}_{1}$.

Now, we shall show that

$$
\left[a_{1}^{\prime}, b_{1}^{\prime}\right] \underset{f}{\longrightarrow}\left[a_{2}^{\prime}, b_{2}^{\prime}\right] \underset{f}{\longrightarrow} \ldots \underset{f}{\longrightarrow}\left[a_{M}^{\prime}, b_{M}^{\prime}\right] \underset{f}{\longrightarrow}\left[a_{1}^{\prime}, b_{1}^{\prime}\right] .
$$

Let us fix $i_{0} \in\{1,2, \ldots, M\}$. Then we have

$$
\begin{aligned}
f\left(\left[a_{i_{0}}^{\prime}, b_{i_{0}}^{\prime}\right]\right) & =f_{i_{0}}\left(\xi_{i_{0}}\left(\left[a_{i_{0}}^{\prime}, b_{i_{0}}^{\prime}\right]\right)\right)+\delta_{i_{0}+1} \\
& \supset\left[a_{i_{0}+1}, b_{i_{0}+1}\right]+\delta_{i_{0}+1}=\left[a_{i_{0}+1}^{\prime}, b_{i_{0}+1}^{\prime}\right],
\end{aligned}
$$

which proves (3).

Let $x$ be an arbitrary point such that $x \in\left[a_{1}^{\prime}, b_{1}^{\prime}\right]$ and $f(x) \in\left[a_{2}^{\prime}, b_{2}^{\prime}\right]$, $f^{2}(x) \in\left[a_{3}^{\prime}, b_{3}^{\prime}\right], \ldots, f^{M-1}(x) \in\left[a_{M}^{\prime}, b_{M}^{\prime}\right], f^{M}(x) \in\left[a_{1}^{\prime}, b_{1}^{\prime}\right]$.

So we have:

$$
\begin{gathered}
f_{1}\left(\xi_{1}(x)\right)=\xi_{2}(f(x)) \\
f_{2}\left(f_{1}\left(\xi_{1}(x)\right)\right)=f_{2}\left(\xi_{2}(f(x))\right)=\xi_{3}\left(f^{2}(x)\right) .
\end{gathered}
$$

Consequently, one can obtain (by induction):

$$
\left(f_{i} \circ\left(f_{i-1} \circ \ldots \circ f_{1}\right)\right)\left(\xi_{1}(x)\right)=f_{i}\left(\xi_{i}\left(f^{i-1}(x)\right)\right)=\xi_{i+1}\left(f^{i}(x)\right) .
$$
3.2 .

This completes the proof of (2) and, at the same time, the proof of Theorem 
As we mentioned in the introduction to this section, the main goal of this part of the paper concentrates on the following problem: What kind of conditions should we impose on $f$ in order to have: there exists $\mathcal{A S}$-ring of Sharkovsky functions containing $f$ ? The partial answer to this question is contained in the following theorem.

Theorem 3.3. Let $f \in \mathcal{S}$. Then $f$ is a Sharkovsky function and there exists an $\mathcal{A S}$-ring $\mathcal{R} \subset \mathcal{S}$ such that $f \in \mathcal{R}$.

Proof. The fact that $f$ is a Sharkovsky function follows immediately from the Lemma 3.2 .

Since $f$ is an $\mathcal{S}$-function, then $f \in \mathcal{S}\left(H,\left\{d_{n}\right\}\right)$, and consequently $f \in$ $\operatorname{FRC}\left(H,\left\{d_{n}\right\}\right)$ with respect to some od-set $H$ and $H$-trajectory $\left\{d_{n}\right\}$.

On the other hand, since $f \in \mathcal{S}\left(H,\left\{d_{n}\right\}\right)$ then for each compact interval $I \subset \mathbb{R}$, there exists an open interval

$$
U_{I} \subset I \cap H \text { such that } \overline{U_{I}} \underset{f}{\longrightarrow} f(I) .
$$

Now, let us establish mutual correspondence $I \leftrightarrow U_{I}$, for each compact interval $I \subset \mathbb{R}$ (i.e. $U_{I}$ is a fixed open interval connected with $I$ such that $U_{I} \subset I \cap H$ and $\left.\overline{U_{I}} \underset{f}{\longrightarrow} f(I)\right)$.

Moreover, if $I \subset \mathbb{R}$ is an arbitrary compact interval and $x \in I$, then let $u_{x}^{I}$ be a fixed element of $\overline{U_{I}}$ such that $f(x)=f\left(u_{x}^{I}\right)$. So, we have established mutual correspondence (one-to-one function)

$$
I \ni x \leftrightarrow u_{x}^{I} \in \overline{U_{I}}
$$

between $x \in I$ and some element $u_{x}^{I}$ belonging to $\overline{U_{I}}$.

Let $\mathcal{R}$ be a family of all functions $g: \mathbb{R} \rightarrow \mathbb{R}$ such that

(A) $g \in \operatorname{FRC}\left(H,\left\{d_{n}\right\}\right)$;

(B) for each compact interval $I$ and any $x \in I, g(x)=g\left(u_{x}^{I}\right)$.

We will show that $\mathcal{R}$ is a required $\mathcal{A S}$-ring. Of course $f \in \mathcal{R}$. For the proof we have to verify axioms of definition of $\mathcal{A S}$-ring. Since the ideas of the proof of Axioms 1-4 are identical then we will present only the proof of Condition 4.

Let $\left\{h_{n}\right\} \subset \mathcal{R}$ and $\left\{h_{n}\right\}$ uniformly converge to $h$. We have to show that $h \in \mathcal{R}$.

First we may observe that $h \in \operatorname{FRC}\left(H,\left\{d_{n}\right\}\right)$. So, let $(a, b)$ be a component of $H, x \in[a, b)$ and $P_{x}^{r}$ be a right first return path to $x$ based on $\left\{d_{n}\right\}$ (of 
course, if $(a, b)$ is halfline or whole line, then the further considerations will be restricted to the suitable cases). Let us denote $\tilde{P}_{x}^{r}=P_{x}^{r} \cup\{x\}$. Then we can consider $\tilde{P}_{x}^{r}$ as a metric space such that $h_{n}\left\lceil\tilde{P}_{x}^{r}\right.$ are continuous functions at $x$ (for $n=1,2, \ldots)$. Then $h \uparrow \tilde{P}_{x}^{r}$ as a uniform limit of $\left\{h_{n} \uparrow \tilde{P}_{x}^{r}\right\}$ is also a continuous function at $x$. This gives

$$
\lim _{\substack{t \rightarrow x \\ t \in P_{x}^{r}}} h(t)=\lim _{\substack{t \rightarrow x \\ t \in P_{x}^{r}}} h \uparrow \tilde{P}_{x}^{r}(t)=h \uparrow \tilde{P}_{x}^{r}(x)=h(x) .
$$

In a similar way we can prove a left-sided first return continuity of $h$ at $x$.

Now, let $I=[\alpha, \beta]$ be an arbitrary compact subinterval of $\mathbb{R}$. Let us fix $x \in I$. According to our assumptions

$$
h_{n}(x)=h_{n}\left(u_{x}^{I}\right), \text { for } n=1,2, \ldots
$$

Let us notice that $h(x)=\lim _{n \rightarrow \infty} h_{n}(x)=\lim _{n \rightarrow \infty} h_{n}\left(u_{x}^{I}\right)=h\left(u_{x}^{I}\right)$. In this way, the verification of Axiom 4. is finished.

According to our requirement connected with functions belonging to the family $\mathcal{R}$ and Theorem 3.2 one can remark that $\mathcal{R} \subset \mathcal{S}\left(H,\left\{d_{n}\right\}\right)$.

Now, we can return to the considerations beginning this section. We have mentioned that the Sharkovsky property is not a stable property (in the sense of [1]). Nonetheless, according to Theorem 3.3, one can observe that for each function $f \in \mathcal{S}$ there exists a family $\mathcal{R}$ of Sharkovsky functions such that $B(f, \epsilon) \cap \mathcal{R} \neq \emptyset$, for any $\epsilon>0$. Consequently, "small perturbations" of $f$ within set (ring) $\mathcal{R}$ preserve the Sharkovsky property.

Theorem 3.3 gives one method for the construction of rings consisting of Sharkovsky functions containing a fixed function $f \in \mathcal{S}$. On the other hand, one can formulate questions connected with the effectiveness of this method. For the answer, notice that even for continuous functions this method permits us to obtain an uncountable family of different rings such that each of them has infinite Goldie dimension ${ }^{4}$. For substantiation of this remark let $f: \mathbb{R} \longrightarrow \mathbb{R}$ be an arbitrary continuous function and let $\left\{\left(a_{n}, b_{n}\right)\right\}$ be a sequence of open intervals such that $\left(a_{n}, b_{n}\right) \searrow 0,1>b_{1}>a_{1}>b_{2}>a_{2}>\ldots$. We will denote by $h_{i}:\left(a_{i}, b_{i}\right) \longrightarrow \mathbb{R}(i=1,2, \ldots)$ a continuous function such that $h_{i}\left(\left(a_{i}, c_{i}\right)\right)=[0,1]=h_{i}\left(\left(c_{i}, b_{i}\right)\right)$, for each $c_{i} \in\left(a_{i}, b_{i}\right)$. Moreover, let $x_{k} \in\left(a_{k}, b_{k}\right)(k=1,2)$. Additionally, we can assume that $h_{2}\left(x_{2}\right)=x_{1}$ and $h_{1}\left(x_{1}\right)=1$. Let us define functions $f_{\alpha}: \mathbb{R} \longrightarrow \mathbb{R}(\alpha \in[0,1])$ in the following

\footnotetext{
${ }^{4} \mathrm{~A}$ family $\left\{\Im_{t}: t \in T\right\}$ of nonzero ideals of some ring $\mathcal{R}$ is said to be independent if $\Im_{t_{0}} \cap\left(\sum_{t \neq t_{0}} \Im_{t}\right)=\left(\text { const }_{0}\right)_{\mathcal{R}}$, for any $t_{0} \in T$. A Goldie dimension of a ring $\mathcal{R}$ (we shall denote by $\operatorname{dim}(\mathcal{R}))$ is the smallest cardinal number $\mathbf{m}$ for which any independent set of nonzero ideals has cardinality less than or equal to $\mathbf{m}$.
} 
way: $f_{\alpha}(x)=0$, if $x \in(-\infty, 0] ; f_{\alpha}(x)=h_{i}(x)$, if $x \in\left(a_{i}, b_{i}\right), i=1,2, \ldots$; $f_{\alpha}(x)=\alpha$, if $x \in\left(0, a_{1}\right) \backslash \bigcup_{i=2}^{\infty}\left(a_{i}, b_{i}\right) ; f_{\alpha}\left\lceil\left[b_{i}, \infty\right)\right.$ is a continuous function such that $f_{\alpha}\left(b_{i}\right)=0$ and $f_{\alpha}(1)=x_{2}$. It is easy to see that $f_{\alpha} \in \mathcal{D} \mathcal{B}_{1}$, for each $\alpha \in[0,1]$ (consequently, according to Proposition 2.3, $f \in \mathcal{S}$ ). Let us put $H=\mathbb{R}$, then $H$ is an od-set and (Proposition 2.3) for any $\alpha \in[0,1]$ there exists an $H$-trajectory $\left\{d_{n}^{\alpha}\right\}$ such that $f_{\alpha} \in \mathcal{S}\left(H,\left\{d_{n}^{\alpha}\right\}\right)$. It is easy to see that $f \in \mathcal{S}\left(H,\left\{d_{n}^{\alpha}\right\}\right)$, too.

Let us consider the method of construction of rings described in the proof of the Theorem 3.3. For each $I \subset \mathbb{R}, x \in I$ and open interval $U_{I}$ connected with $I$, let us put $u_{x}^{I}=x$. Then ring $\mathcal{R}$ depends only on $\left\{d_{n}^{\alpha}\right\}$. In this way we can construct rings $\left\{\mathcal{R}_{\alpha}\right\}_{\alpha \in[0,1]}$ such that $f_{\alpha} \in \mathcal{R}_{\alpha}, f \in \mathcal{R}_{\alpha}$, for $\alpha \in[0,1]$. If $\beta \neq \alpha$, then $f_{\alpha}-f_{\beta} \notin \mathcal{D}$, and (Theorem 2.2) $f_{\beta} \notin \mathcal{R}_{\alpha}$ and, consequently, $\mathcal{R}_{\alpha} \neq \mathcal{R}_{\beta}$, for $\alpha \neq \beta$. It remained to show that $\operatorname{dim}\left(\mathcal{R}_{\alpha}\right) \geq \aleph_{0}$.

Before proceeding further, we shall introduce some notation. For every $i=1,2 \ldots$, let $a^{i}, b^{i}, c^{i}$ denote points belonging to $\left(a_{i}, b_{i}\right)$ such that $a_{i}<a^{i}<$ $c^{i}<b^{i}<b_{i}$. Let $g_{i}$ be a continuous function $(i=1,2 \ldots)$ such that $g_{i}\left(c^{i}\right)=1$; $g_{i}(x)=0$, if $x \in\left[0, a^{i}\right] \cup\left[b^{i}, 1\right]$. Of course, $g_{i} \in \mathcal{R}_{\alpha}$, for $i=1,2, \ldots$ and $\alpha \in[0,1]$.

Let us fix $\alpha_{0} \in[0,1]$. Now, we can consider the family of ideals generated by functions $g_{i}:\left\{\left(g_{i}\right): i=1,2, \ldots\right\}$. It is sufficient to show that $\left\{\left(g_{i}\right): i=\right.$ $1,2, \ldots\}$ is a family of independent ideals.

In fact. Let $i_{0}$ be a fixed positive integer. Then $g_{i_{0}} \cdot g_{i}=$ const $_{0}$, for any $i=1,2, . ., i_{0}-1, i_{0}+1, \ldots$ So let $h \in\left(g_{i_{0}}\right) \cap \sum_{i \neq i_{0}}\left(g_{i}\right)$. Note that $h=\mu \cdot g_{i_{0}}$ for some $\mu \in \mathcal{R}_{\alpha_{0}}$ and, on the other hand,

$$
h=\mu_{k_{1}} \cdot g_{k_{1}}+\ldots+\mu_{k_{m}} \cdot g_{k_{m}}
$$

for some positive integers $k_{1}, \ldots, k_{m}$ and $\mu_{k_{1}}, \ldots, \mu_{k_{m}} \in \mathcal{R}_{\alpha_{0}}$. Then $h^{2}=$ const $_{0}$. Consequently, $\left(g_{i_{0}} \cap \sum_{i \neq i_{0}}\left(g_{i}\right)\right)=\left(\right.$ const $\left._{0}\right)$, and so $\left\{\left(g_{i}\right): i=1,2, \ldots\right\}$ is a family of independent ideals.

Despite the fact that Theorem 3.3 shows that for the rather wide class of Sharkovsky one can find a ring consisting of Sharkovsky functions containing a fixed function belonging to this family, in general, the following question is still unsolved:

Problem 1. What kind of conditions characterize a family $\mathcal{F}$ consisting of all Sharkovsky functions $f$ for which there exists an AS-ring (or usual ring) of Sharkovsky functions containing $f$ ?

However, it is easy to construct a function $f \in \mathcal{S}$ and some $\mathcal{A S}$-ring $\mathcal{R}$ of $\mathcal{S}$-functions, containing $f$ such that the identical function does not belong to $\mathcal{R}$.

The above considerations lead us to the following question. 
Problem 2. Let $f$ be a fixed $\mathcal{S}$-function. Characterize continuous functions belonging to either each or some $\mathcal{A S}$-ring of $\mathcal{S}$-functions containing $f$.

\section{References}

[1] L. Alsedà, J. Llibre, M. Misiurewicz, Combinatorial dynamics and entropy in dimension one, Advanced Series in Nonlinear Dynamics, 5, World Scientific Publishing, River Edge, NJ, 1993.

[2] G. Aumann, Reelle Funktionen, Berlin - Götingen - Heidelberg, 1954.

[3] L. S. Block, W. A. Coppel, Dynamics in one dimension, Lecture Notes in Mathematics, 1513, Springer-Verlag, Berlin, 1992.

[4] A. M. Bruckner, Differentiation of real functions, Second edition, CRM Monograph Series, 5, American Mathematical Society, Providence, RI, 1994.

[5] A. M. Bruckner, J. G. Ceder, On the sum of Darboux functions, Proc. Amer. Math. Soc., 51(1) (1975), 97-102.

[6] K. Ciesielski, Set theoretic real analysis, J. Appl. Anal., 3(2) (1997), 143190.

[7] M. Čiklová, Dynamical systems generated by functions with connected $G_{\delta}$ graphs, Real Anal. Exchange, 30(2) (2004-2005), 617-638.

[8] I. Ćwiek, R. J. Pawlak, Świątek B., On some subclasses of Baire 1 functions, Real Anal. Exchange, 27(2) (2001-2002), 415-422.

[9] U. B. Darji, M. J. Evans, R. J. O'Malley, A first return characterization of Baire one functions, Real Anal. Exchange, 19 (1993-1994), 510-515.

[10] U. B. Darji, M. J. Evans, R. J. O'Malley, First return path systems: differentiability, continuity and orderings, Acta Math. Hungar., 66(1-2) (1995), 83-103.

[11] U. B. Darji, M. J. Evans, R. J. O'Malley, Universally first return continuous function, Proc. Amer. Math. Soc., 123(9) (1995), 2677-2685.

[12] U. B. Darji, M. J. Evans, P. D. Humke, First return approachability, J. Anal. Appl., 199 (1996), 545-557. 
[13] R. Devaney, An Introduction to Chaotic Dynamical Systems, Second edition, Addison-Wesley Studies in Nonlinearity, Addison-Wesley Publishing, Advanced Book Program, Redwood City, CA, 1989.

[14] R. Engelking, General Topology, Translated from the Polish by the author, Monografie Matematyczne, 60 [Mathematical Monographs, 60] PWN-Polish Scientific Publishers, Warsaw, 1977.

[15] M. J. Evans, R. J. O'Malley, First return limiting notions in real analysis, Real Anal. Exchange, 29(2) (2003-2004), 503-530.

[16] R. Fleissner, A note on Baire 1 Darboux functions, Real Anal. Exchange, 3 (1977-1978), 104-106.

[17] Z. Grande, On the Darboux property of the sum of cliquish functions, Real Anal. Exchange, 17(2) (1991-1992), 571-576.

[18] Z. Grande, On the sums and products of Darboux Baire*1 functions, Real Anal. Exchange, 18(1) (1991-1992), 237-240.

[19] J. Jastrzębski, J. Jȩdrzejewski, T. Natkaniec, On some subclasses of Darboux functions, Fund. Math., 138 (1991), 165-173.

[20] K. R. Kellum, Sums and limits of almost continuous functions, Colloq. Math., 31 (1974), 125-128.

[21] K. L. Kellum, Iterates of almost continuous functions and Sharkovskii's theorem, Real Anal. Exchange, 14 (1988-89), 420-422.

[22] E. Korczak-Kubiak, Pierścienie funkcji H-spójnych, Doctoral Thesis, Ldz University, 2009.

[23] A. Maliszewski, Darboux property and quasi-continuity. Uniform approach, Habilitation Thesis, Pedagogical University Słupsk, 1996.

[24] T. Natkaniec, On compositions and products of almost continuous functions, Fund. Math., 139 (1991), 71-78.

[25] T. Natkaniec, Almost continuity, Habilitation Thesis, Pedagogical University, Bydgoszcz, 1992.

[26] H. Pawlak, R. J. Pawlak, Transitivity, dense orbits and some topologies finer than the natural topology of the unit interval, (English summary), Tatra Mt. Math. Publ., 35 (2007), 1-12. 
[27] R. J. Pawlak, On rings of Darboux functions, Colloq. Math., 53 (1987), 289-300.

[28] R. J. Pawlak, On complete systems of almost continuous functions, Tatra Mt. Math. Publ., 5 (1994), 51-59.

[29] R. J. Pawlak, On the entropy of Darboux functions, Colloq. Math., (to appear).

[30] R. J. Pawlak, On the Sharkovsky's property of Darboux functions, Tatra Mt. Math. Publ., (to appear).

[31] A. Peris, Transitivity, dense orbit and discontinuous functions, Bull. Belg. Math. Soc., 6 (1999), 391-394.

[32] P. Szuca, Punkty state odwzorowań typu Darboux, (Polish), Doctoral Thesis, Gdańsk, 2003.

[33] P. Szuca, Sharkovskii's theorem holds for some discontinuous functions, Fund. Math., 179 (2003), 27-41.

[34] A. Tomaszewska, On the set of functions possessing the property (top) in the space on Darboux and Światkowski functions, Real Anal. Exchange, 19(2) (1993-1994), 465-470. 
H. PAWlak AND R. J. PAWlak 